\title{
THE EMERGENCE OF WEAKLY TWISTED MAGNETIC FIELDS IN THE SUN
}

\author{
V. ARChONTIS ${ }^{1,2}$, A. W. HoOd ${ }^{2}$, AND K. TsinganOs ${ }^{3}$ \\ ${ }^{1}$ Section of Astrophysics, Astronomy and Mechanics, Department of Physics, University of Athens, \\ Panepistimiopolis 15784, Athens, Greece \\ ${ }^{2}$ School of Mathematics and Statistics, St. Andrews University, St. Andrews KY169SS, UK \\ ${ }^{3}$ National Observatory of Athens, Lofos Nymphon, Thissio 11810, Athens, Greece \\ Received 2013 January 22; accepted 2013 September 9; published 2013 November 1
}

\begin{abstract}
We have studied the emergence of a weakly twisted magnetic flux tube from the upper convection zone into the solar atmosphere. It is found that the rising magnetized plasma does not undergo the classical, single $\Omega$-shaped loop emergence, but it becomes unstable in two places, forming two magnetic lobes that are anchored in small-scale bipolar structures at the photosphere, between the two main flux concentrations. The two magnetic lobes rise and expand into the corona, forming an overall undulating magnetic flux system. The dynamical interaction of the lobes results in the triggering of high-speed and hot jets and the formation of successive cool and hot loops that coexist in the emerging flux region. Although the initial emerging field is weakly twisted, a highly twisted magnetic flux rope is formed at the low atmosphere, due to shearing and reconnection. The new flux rope (hereafter post-emergence flux rope) does not erupt. It remains confined by the overlying field. Although there is no ejective eruption of the post-emergence rope, it is found that a considerable amount of axial and azimuthal flux is transferred into the solar atmosphere during the emergence of the magnetic field.
\end{abstract}

Key words: Sun: activity - Sun: magnetic fields

Online-only material: color figures

\section{INTRODUCTION}

The emergence of magnetic flux from the solar interior is a multi-scale process that has been repeatedly observed in the Sun (e.g., Leka et al. 1996; Centeno et al. 2007; Vargas Domínguez et al. 2012b), and it is thought to be responsible for the onset of various dynamic phenomena, such as the formation of active regions (ARs; e.g., Liggett \& Zirin 1985; Zwaan 1985), the creation of jets (e.g., Shibata et al. 1992; Canfield et al. 1996), and the triggering of eruptions (e.g., van Driel-Gesztelyi \& Culhane 2009; Schrijver 2009).

A series of numerical experiments have been invoked to study the aforementioned phenomena, based on the rising motion of a buoyant, twisted magnetic flux tube or flux sheet from the solar interior into the corona. In common twisted flux tube models (e.g., Fan 2001; Magara \& Longcope 2003; Archontis et al. 2005), the fieldlines are winding about the axis of the tube, through an angle $\alpha L$, over the length $L$ of the tube in a uniform manner. A measure of the twist per unit length is given by the parameter $\alpha$. For instance, a value of $\alpha=22 \times 10^{-4} \mathrm{~km}^{-1}$ makes the tube marginally stable to the kink instability; thus, we refer to this as the strongly twisted case. On the other hand, in the present work (see Section 2.2), we use a small value of $\alpha=5.5 \times 10^{-4} \mathrm{~km}^{-1}$, for which a magnetic flux tube with $\mathrm{\iota}=36 \mathrm{Mm}$ possesses a low level of twist (only three full turns over $L$ ), and we refer to it as the weakly twisted case.

Although the initial distribution of the magnetic field in the aforementioned simulations is represented by a rather monolithic magnetic flux structure, these models have been successful in reproducing the dynamics of various solar events such as the filamentary structure in emerging flux regions (EFRs; Isobe et al. 2005), the emission of outflows/jets at the edges of ARs (Gontikakis et al. 2009; Harra et al. 2012), the complex nature of sigmoidal regions (Archontis et al. 2009), the topology and dynamics of jets (e.g., Yokoyama \& Shibata 1995; MorenoInsertis et al. 2008; Baumann \& Nordlund 2012), and the largescale evolution of the field in EFRs and ARs.

A key parameter in these experiments is the degree of twist of the emerging field. The appearance of twisted magnetic field structures in EFRs has been shown by early observations (e.g., Leka et al. 1996). Also, the appearance of magnetic tails or magnetic tongues in observations of ARs (e.g., López Fuentes et al. 2000; Canou \& Amari 2010; Luoni et al. 2011) and numerical experiments (Archontis \& Hood 2010) has been interpreted as direct evidence of an emerging field with a strongly azimuthal nature. On the other hand, it has been found that in many EFRs the topology of the magnetic field adopts a "sea serpent" configuration (e.g., Pariat et al. 2004), which does not necessarily imply that the emerging field encompasses a large amount of twist. The question of whether the emerging fields are already (weakly or strongly) twisted or become twisted during their rising motion within the solar convection zone is still unanswered.

Convection zone simulations have shown that in order for the magnetic field to remain coherent during its rising motion, a minimum amount of twist is needed (Moreno-Insertis \& Emonet 1996; Abbett et al. 2000). However, it has also been shown that the convective disruption and dynamic evolution of a flux tube do not depend critically on the initial amount of the twist (Fan et al. 2003) if the strength of the field is greater than a certain value (e.g., $3 B$, where $B$ is the field strength corresponding to the kinetic energy density of strong downdrafts in the convection zone). In the latter case, the rising magnetic field can even modify the granulation pattern (e.g., Cheung et al. 2007). Otherwise, the magnetic field becomes susceptible to distortion by convection and may naturally develop a "sea serpent" configuration due to small-scale flux emergence driven by granular convection (e.g., Cheung et al. 2008; TortosaAndreu \& Moreno-Insertis 2009; Bushby \& Archontis 2012). 
In more idealized simulations, it has been shown that the undulating shape of the emerging magnetic field (e.g., a flux sheet) at the photosphere develops due to the excitation of the Parker instability (Isobe et al. 2007), and there are observations (Otsuji et al. 2011) that support this scenario. Also, parametric studies (Toriumi \& Yokoyama 2011) have demonstrated that magnetic fields in the deep convection zone $(\approx 20 \mathrm{Mm}$ below the surface) should have a field strength of at least $B=1.5 \times 10^{4} \mathrm{G}$ and twist larger than $2.5 \times 10^{-4} \mathrm{~km}^{-1}$ in order to emerge at the surface. Otherwise, they experience a failed emergence and remain within the convection zone. The accumulation of magnetic flux at the photosphere without further emergence into the corona has also been reported in experiments (Murray et al. 2006) where a flux tube starts to rise from the upper convection zone (at $\approx 1.7 \mathrm{Mm}$ below the surface), with a twist of $5.8 \times 10^{-4} \mathrm{~km}^{-1}$ and a field strength of $B=3.8 \times 10^{3} \mathrm{G}$.

The actual emergence of a twisted magnetic field as a whole, at the photosphere and above, has also been investigated in previous studies. Observations (Lites et al. 2010) of ARs using the Solar Optical Telescope on board Hinode and Transition Region and Coronal Explorer have suggested (although not conclusively) that a twisted magnetic field can emerge bodily and form an AR filament channel. Similar observations (Okamoto et al. 2008) have reported on the emergence of a helical flux rope from below the photosphere into the corona, along the polarity inversion line (PIL) of an AR. These observations have been discussed in the work by Vargas Domínguez et al. (2012a), who interpreted the photospheric behavior along the PIL to be indicative of magnetic flux cancellation driven by flows from the dominant sunspot of the AR. In addition, numerical experiments (e.g., Manchester et al. 2004; Gibson \& Fan 2006; MacTaggart \& Hood 2009a, 2010; Archontis \& Török 2008; Archontis \& Hood 2012) have suggested that magnetic fields often experience partial emergence, during which a fraction of the magnetic system manages to emerge into the corona while a considerable amount of axial field remains at the low photosphere.

In this paper, we show that the emergence of a weakly twisted (i.e., $5.5 \times 10^{-4} \mathrm{~km}^{-1}$ ) and low plasma- $\beta$ flux tube from the upper convection zone into the solar atmosphere is possible. It occurs primarily through the emergence and expansion of two magnetic lobes above the solar surface. The interaction of the two lobes leads to the onset of other dynamic phenomena within the EFR. In Section 2, we present the initial conditions for the magnetic field and the stratified atmosphere. The instability criterion for the emergence at the photosphere and the topology of the field at the solar surface are considered in Section 3. The emergence above the photosphere and the onset of dynamic phenomena due to the interaction between the magnetic fields within the EFR is examined in Section 4. The transfer of flux into the solar atmosphere during the emergence of the field is presented in Section 5. Section 6 gives a brief discussion and description of the results.

\section{METHOD}

\subsection{Equations}

To perform the required numerical experiments, we solve the three-dimensional (3D) time-dependent, resistive, and compressible MHD equations in Cartesian geometry. The basic equations (here in dimensionless form) are

$$
\frac{\partial \rho}{\partial t}+\nabla \cdot(\rho \mathbf{v})=0
$$

$$
\begin{gathered}
\frac{\partial(\rho \mathbf{v})}{\partial t}=-\nabla \cdot(\rho \mathbf{v v})+(\nabla \times \mathbf{B}) \times \mathbf{B}-\nabla P+\rho \mathbf{g}+\nabla \cdot \mathbf{S} \\
\frac{\partial(\rho \epsilon)}{\partial t}=-\nabla \cdot(\rho \epsilon \mathbf{v})-P \nabla \cdot \mathbf{v}+Q_{\text {Joule }}+Q_{\text {visc }} \\
\frac{\partial \mathbf{B}}{\partial t}=\nabla \times(\mathbf{v} \times \mathbf{B})+\eta \nabla^{2} \mathbf{B}
\end{gathered}
$$

with specific energy density

$$
\epsilon=\frac{P}{(\gamma-1) \rho}
$$

and the ideal gas law

$$
P=\frac{\rho T}{\tilde{\mu}} .
$$

$\mathbf{B}, \rho, P$, and $\mathbf{v}$ stand for the magnetic field vector, the density, the gas pressure, and the velocity vector, respectively. Gravity is included, with $\mathbf{g}=-g \hat{\mathbf{z}}$ being the gravitational acceleration and $g=274 \mathrm{~m} \mathrm{~s}^{-2}$. For the explicit dimensionless resistivity we use a constant value of $\eta=10^{-3}$. The ratio of specific heats is $\gamma$, and $\tilde{\mu}$ is the mean molecular weight. The medium is assumed to be an inviscid, perfect gas with $\gamma=5 / 3$. We also assume that the gas is only weakly ionized $(\tilde{\mu} \approx 1)$. Viscous and Ohmic heating are considered through the viscosity and Joule dissipation $Q_{\text {Joule }}=\eta j^{2}$. The viscous heating term is $Q_{\text {visc }}=\epsilon_{\mathrm{ij}} S_{\mathrm{ij}}$, where $\epsilon_{\mathrm{ij}}$ is the strain rate and $S_{\mathrm{ij}}$ is the viscous stress tensor, similar to the work by Arber et al. (2007). The above equations were numerically solved by using the Lare $3 d$ code (Arber et al. 2001).

To convert into dimensional variables, we use the following units: density $\rho=1.67 \times 10^{-7} \mathrm{~g} \mathrm{~cm}^{-3}$, temperature $T=$ $5100 \mathrm{~K}$, and pressure $P=7.16 \times 10^{4} \mathrm{erg} \mathrm{cm}^{-3}$, where the non-dimensionalization is $P=\rho R T$. For the length, we use $H=180 \mathrm{~km}$ and for the magnetic field strength $B=300 \mathrm{G}$. Using the above units, we obtain the velocity $v=2.1 \mathrm{~km} \mathrm{~s}^{-1}$ and time $t=85.7 \mathrm{~s}$.

\subsection{Initial Conditions}

The initial conditions in our model consist of the background hydrostatic atmosphere and a horizontal weakly twisted magnetic flux tube below the photosphere.

Figure 1 (top panel) shows the initial distribution of the temperature $(T)$, density $(\rho)$, and gas pressure $(P)$ as functions of height. Hydrostatic equilibrium is assumed for the initial atmosphere. The sub-photospheric layer is represented by an adiabatically stratified layer in the range $-3.6 \mathrm{Mm} \leqslant z<$ $0 \mathrm{Mm}$. The photosphere/chromosphere is represented by a layer at $0 \mathrm{Mm} \leqslant z<2.4 \mathrm{Mm}$, which is isothermal at the beginning, and then the temperature increases with height, up to $\approx 4 \times 10^{4} \mathrm{~K}$. The layer above, at $2.4 \mathrm{Mm} \leqslant z \leqslant 3 \mathrm{Mm}$, is mimicking the transition region. The uppermost layer $(3 \mathrm{Mm}<z \leqslant 47 \mathrm{Mm})$ is an isothermal layer that represents the corona. In the corona, the plasma is fully ionized and $\tilde{\mu} \approx 0.5$. Here gravity is not so important. However, in the photosphere, the gas is only weakly ionized and the mean molecular weight in the gas law can be approximated by $\tilde{\mu} \approx 1$. This is the region where gravitational stratification significantly reduces the pressure and density with height. Rather than solve for the ionization fraction (which depends on temperature) and hence for $\tilde{\mu}$, we follow the common approach of $\tilde{\mu} \approx 1$. Note that the temperature does not explicitly appear in the numerical equations and is only determined for the gas law, as expressed in Equation (6).

For the initial magnetic field, we considered a horizontal cylindrical magnetic flux tube (bottom panel of Figure 1), 

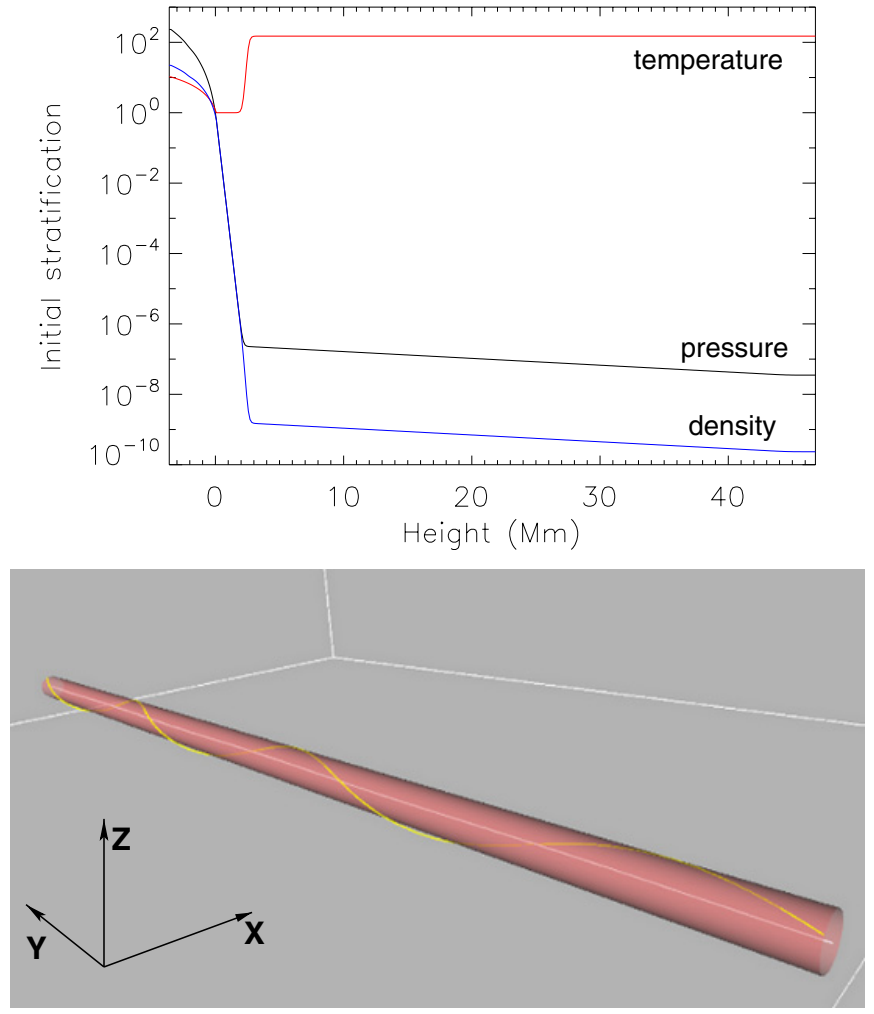

Figure 1. Top: the (dimensionless) stratification of the initial atmosphere: temperature (red), pressure (black), and density (blue). Bottom: the initial magnetic flux tube is visualized by the red transparent isosurface, which represents a field strength of $B=1300 \mathrm{G}$. The yellow fieldline shows the twist at the outermost layers of the tube, while the white fieldline is traced from the axis of the tube.

(A color version of this figure is available in the online journal.)

which is located $2.1 \mathrm{Mm}$ below the photosphere. The flux tube is oriented with its axis along the positive $y$-direction. The transverse direction is $x$, which is predominantly aligned along the east-west direction, and the vertical direction is $z$. The axial field, $B_{y}$, is defined by

$$
B_{y}=B_{0} \exp \left(-r^{2} / R^{2}\right)
$$

and the azimuthal field by

$$
B_{\phi}=\alpha r B_{y},
$$

where $R$ is a measure of the radius of the tube and $r$ is the radial distance from the tube axis. In the following, we choose $R=2.5$ (i.e., $450 \mathrm{~km}$ ). Owing to the above formulation, the field strength decreases with $r$ from the axis following a Gaussian profile.

We choose a uniform twist with $\alpha=5.5 \times 10^{-4} \mathrm{~km}^{-1}$, which implies that we study the evolution of a weakly twisted magnetic flux system. To initiate the rising motion of the tube, we apply the same density distribution as in previous studies (e.g., Archontis $\&$ Hood 2012) that makes the middle part of the tube underdense and, hence, more buoyant. The density deficit is

$$
\Delta \rho=\left[p_{t}(r) / p(z)\right] \rho(z) \exp \left(-y^{2} / \lambda^{2}\right),
$$

where $p_{t}$ is the pressure within the flux tube and is defined as $p+p_{e}$ ( $p$ is the external gas pressure and $p_{e}$ is the pressure excess). Given the field in Equations (7) and (8), we require

$$
p_{e}=\frac{1}{2}\left(\alpha^{2}\left(\frac{R^{2}}{2}-r^{2}\right)-1\right) B_{y}^{2},
$$

for pressure balance.
In Equation (9), $\lambda$ is the approximate length of the buoyant part of the tube. In the following, we use $\lambda=10$ (i.e., $1.8 \mathrm{Mm}$ ) and $B=9.4$ (i.e., $2.8 \mathrm{kG}$ ) for the initial field strength of the sub-photospheric flux tube. For this field strength, the plasma $\beta$ is approximately 25 at the center of the flux tube, well above unity.

For the computational domain, we assume periodic boundary conditions in the $x$ - and $y$-directions. At the top boundary, we include a wave damping zone for $z>43 \mathrm{Mm}$ where fluctuations of velocity, gas pressure, and density decay exponentially with height. The numerical domain has a size of $[-25.2,25.2] \times[-25.2,25.2] \times[-3.6,47] \mathrm{Mm}$ in the longitudinal $(y)$, transverse $(x)$, and vertical $(z)$ directions, respectively.

\section{EMERGENCE AT THE PHOTOSPHERE}

\subsection{Two-loop Emergence and Fieldline Topology}

Figure 2 shows the distribution of the vertical magnetic field (at $z=0 \mathrm{Mm}, z=0.54 \mathrm{Mm}$ ) and the topology of the fieldlines, at two different times (left and right columns in Figure 2). Due to the density deficit, the apex of the buoyant part of the tube rises and intersects the base of the photosphere, forming a single bipolar structure in a north-south orientation (i.e., along the transverse direction) due to the azimuthal nature of the field at the top. Eventually, the two polarities (hereafter, main polarities) move apart toward an east-west orientation (i.e., along the $y$-axis) and the magnetic field spreads horizontally due to the isothermal stratification of the photosphere.

At $z=0 \mathrm{Mm}$, the most pronounced feature is the appearance of two opposite polarities of magnetic flux. Between them, the magnetic field strength is small and the field is mainly horizontal due to the small twist. Thus, the field does not possess striking, strong vertical $B$-field tails (as in the case of strongly twisted emerging flux tubes) but rather consists of weakly twisted fieldlines that form a horizontal flux sheet (e.g., white lines in Figure 2) that join directly the two main polarities. At slightly larger heights (e.g., $z=0.54 \mathrm{Mm}$, middle left panel) there is no trace of magnetic tails. The rising magnetic field appears to intersect the surface in four places, adopting a double bipolar configuration. Initially, the orientation of the two bipoles makes a small angle with respect to the $y$-axis, but eventually they undergo an anticlockwise rotation turning into an east-west orientation following the general evolution of the system.

The blue (red) fieldlines in Figure 2 have been traced from the positive (negative) polarity of the left (right) lobe at $z=0.54 \mathrm{Mm}$. The top view panels at $t=140$ minutes show that these fieldlines are basically oriented along the east-west direction and that they are ultimately anchored in the two main polarities (at $z=0 \mathrm{Mm}$ ) of the emerging field. The bottom side view panel at $t=140$ minutes reveals that segments of these fieldlines at the sites of the two bipoles have just started to emerge. Note that the two bipolar structures are not necessarily joined by the same set of fieldlines. Eventually, this emergence forms two magnetic flux systems (hereafter, magnetic lobes), which rise further into the solar atmosphere.

Indeed, at a later time ( $t=154$ minutes), the fieldlines of the two lobes expand into the 3D space, adopting a fan-like shape. The expansion is driven by the magnetic pressure, which overwhelms the outside gas pressure that decreases rapidly with height. Now the lobes are joined with the same fieldlines that posses $U$-shaped dips at the low photosphere: blue and red fieldlines are passing through both bipoles (see Figure 2, bottom 

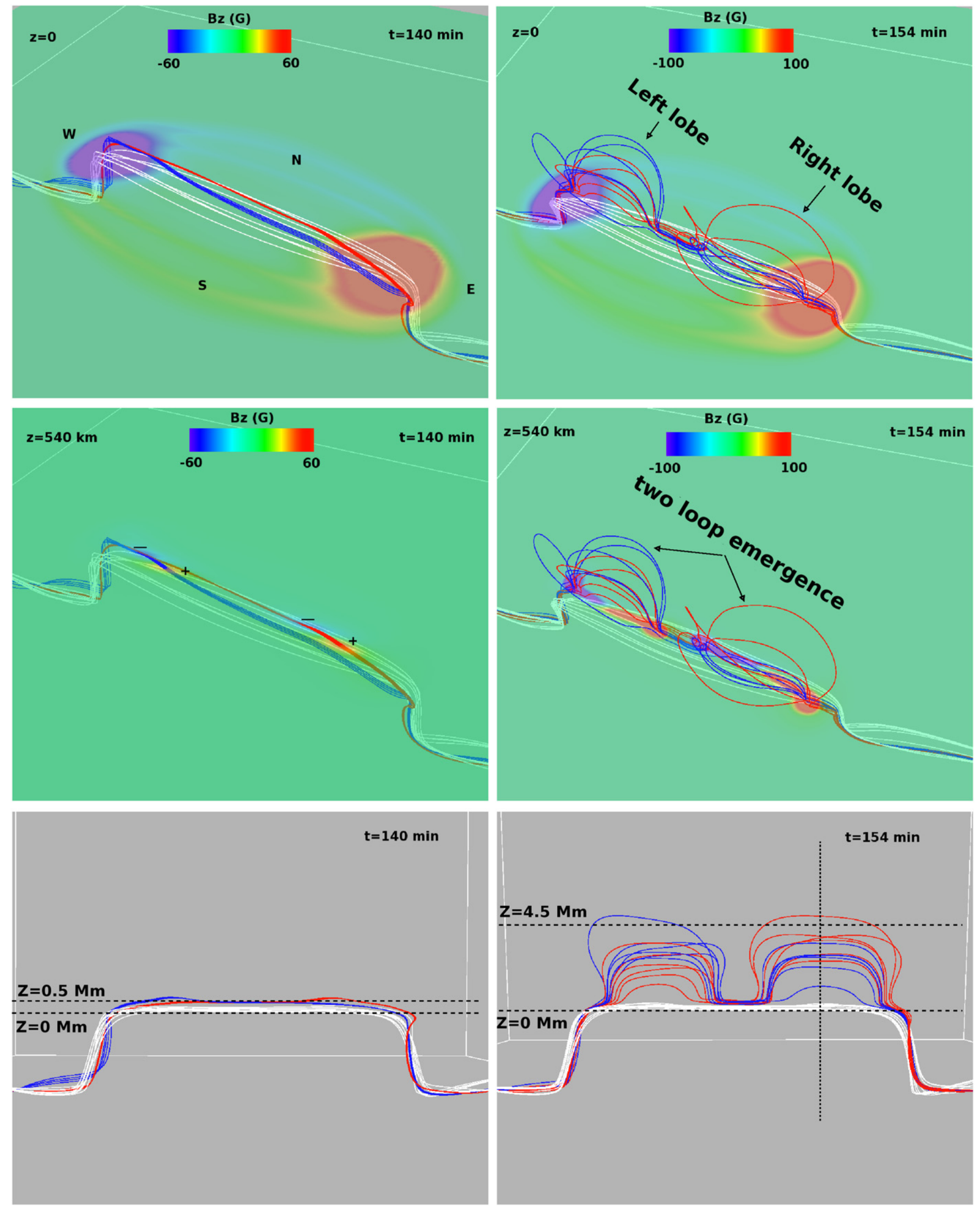

Figure 2. Top: $B_{\mathrm{Z}}$ distribution at the base of the photosphere at two different times. Middle: the same as above, at $z=0.54 \mathrm{Mm}$. Bottom: side view of the fieldlines at the same times. The dashed vertical line is located at the center of the right lobe.

(A color version of this figure is available in the online journal.) 
right). Thus, an overall undulating magnetic flux system has been formed, although the initial sub-photospheric flux tube was straight and horizontal. It is worthwhile mentioning that the white fieldlines, which are located close to the main axis of the flux tube, remain at the low photosphere (not above 2-3 local pressure scale heights), while the lower segments ( $U$-shaped) of the emerging fieldlines lie above them. Similar results were shown in previous studies (e.g., Archontis \& Hood 2010) that investigated the effect of twist on the emergence of the field. It was demonstrated that the emergence of weakly twisted fields at the photosphere leads to a complex distribution of magnetic flux that adopts an undulating configuration.

\subsection{The Onset of the Buoyancy Instability}

As in previous studies (e.g., Archontis et al. 2004), we use the instability criterion by Acheson (1979) to investigate the further emergence of the field above the photosphere. More precisely, we apply the following critical condition:

$$
-H_{p} \frac{\partial}{\partial z}(\log B)>-\frac{\gamma}{2} \beta \delta+{\tilde{k_{\|}}}^{2}\left(1+\frac{{\tilde{k_{\perp}}}^{2}}{{\tilde{k_{z}}}^{2}}\right),
$$

where the pressure scale height is $H_{p}$, the magnitude of the magnetic field vector, $B$, the ratio of specific heats, $\gamma$, the plasma$\beta$, and perturbations with wavevector $\mathbf{k}$, where $\tilde{k_{\|}}$and $\tilde{k_{\perp}}$ are the horizontal components parallel and perpendicular to the magnetic field and $\tilde{k}_{z}$ is the vertical component. The wavevector components are calculated using the horizontal widths, $l_{x}$ and $l_{y}$, and the radius of the tube that approximately stands for $l_{z}$. An estimate of the horizontal widths when the emergence of the magnetic lobes starts (e.g., at $t=137$ minutes and $z=0.36 \mathrm{Mm})$ shows that $l_{y} \approx 80.952$ and $l_{x} \approx 36.9048$. Accordingly, the wavevector components become $\tilde{k_{\|}} \approx 0.077$, $\tilde{k_{\perp}} \approx 0.17$, and $\tilde{k_{z}} \approx 2.52$. The superadiabatic excess, $\delta$, is given by $\delta=\nabla-\nabla_{\text {ad }}$ and is equal to -0.4 for the isothermal photosphere. $\nabla$ is the actual logarithmic temperature gradient in the equilibrium stratification, and $\nabla_{\mathrm{ad}}$ is its adiabatic value.

Since the term ${\tilde{k_{\|}}}^{2}\left(1+\frac{{\tilde{k_{\perp}}}^{2}}{{\tilde{k_{z}}}^{2}}\right)$ is very small, Equation (11) can be approximated with the following condition:

$$
\frac{\left(-H_{p} \frac{\partial}{\partial z}(\log B)\right)}{\left(-\frac{\gamma}{2} \beta \delta\right)>1}
$$

For simplicity, we call the term on the left-hand side of Equation (12) the instability term. Figure 3 shows the time evolution of this term at the center of the EFR (top panel) and through the center of the right lobe (bottom panel).

We find that the instability term remains small $(<1)$ and, therefore, the field does not become unstable at the center of the EFR. On the other hand, the magnetic field around the lobe meets the condition for instability at the domain $0.36 \mathrm{Mm}<z \leqslant 0.9 \mathrm{Mm}(t=137$ minutes $)$. This domain becomes larger at successively later times; thus, the whole plasma in the close neighborhood is able to emerge above the photosphere. The same evolution is apparent in both lobes.

The above result suggests that when the initial twist of the rising magnetic field is small, the magnetized plasma undergoes a two-loop emergence. By two-loop emergence, we mean the rising motion of the two magnetic lobes at the sides of the EFR. At the center of the EFR, the magnetic field does not become strong enough to exert a force capable of driving further
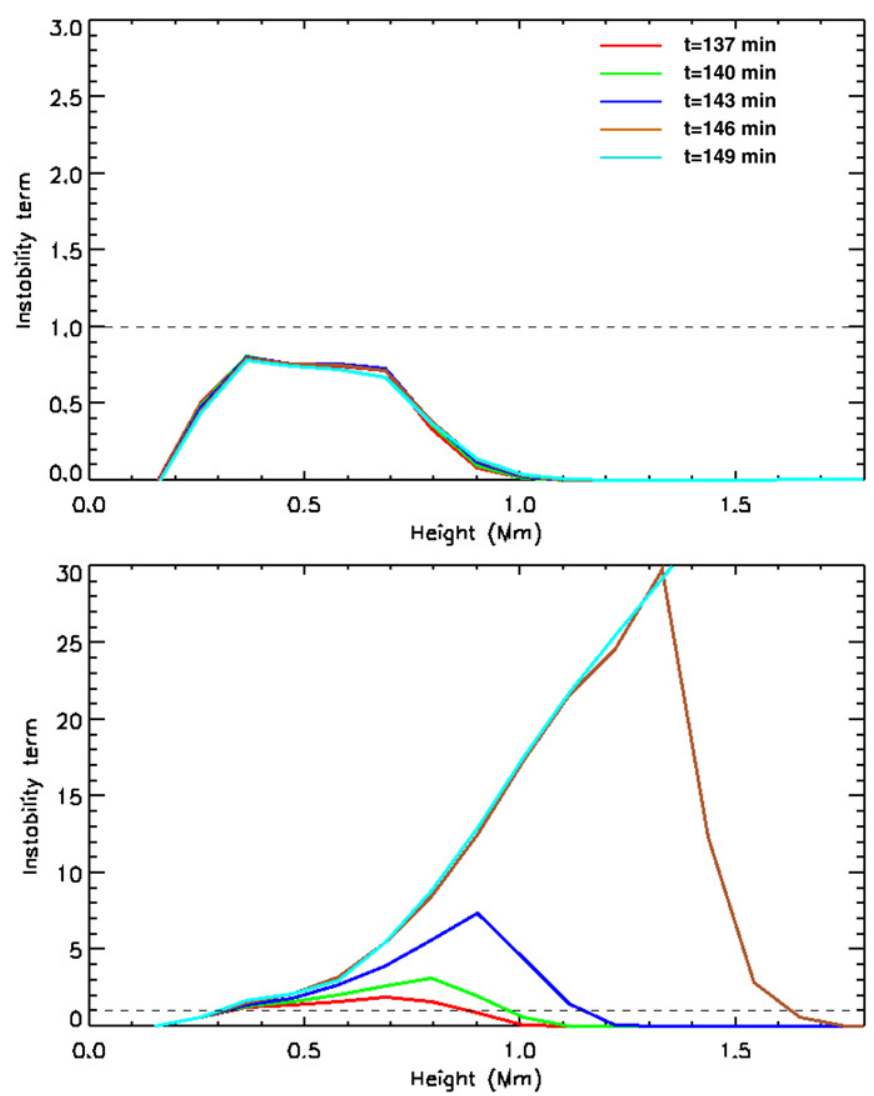

Figure 3. Top: the distribution of the instability term (Equation (12)) along height at the middle of the $\operatorname{EFR}(x=0 \mathrm{Mm}, y=0 \mathrm{Mm})$, at $t=137$ minutes (red), $t=140$ minutes (green), $t=143$ minutes (blue), $t=146$ minutes (orange), and $t=149$ minutes (cyan). Bottom: the same as above, through the center of the right lobe at $x \approx 3.7 \mathrm{Mm}$ and $y=0 \mathrm{Mm}$ (also identified with the vertical dashed line in the bottom right panel in Figure 2).

(A color version of this figure is available in the online journal.)

emergence above the photosphere and possible expansion into the corona. The horizontal spreading of the field aggravates this situation, since the emerging magnetized plasma is moving toward the main polarities and, thus, the magnetic field weakens at the center of the EFR. Consequently, the two-loop emergence occurs naturally as more magnetic layers rise from below and the plasma- $\beta$ reduces to sufficiently small values at sites with strong magnetic field.

\section{INTERACTION BETWEEN THE MAGNETIC LOBES}

An interaction between the magnetic lobes arises spontaneously from the expansion of the field. During the evolution, the central polarities of the lobes (i.e., the negative polarity of the right-side lobe and the positive polarity of the left-side lobe) come closer together, while the side polarities (i.e., the positive polarity of the right-side lobe and the negative polarity of the left-side lobe) move apart and toward the two main polarities. In the following, we describe the plasma response due to the dynamical evolution of the field at the close vicinity between the central polarities of the magnetic lobes.

\subsection{Plasma Response and the Formation of a Post-emergence Flux Rope}

Figure 4 (top and middle panels) shows the density distribution at the vertical $y z$-midplane during the initial stage of the interaction. At $t=151$ minutes, the two-loop emergence of the 

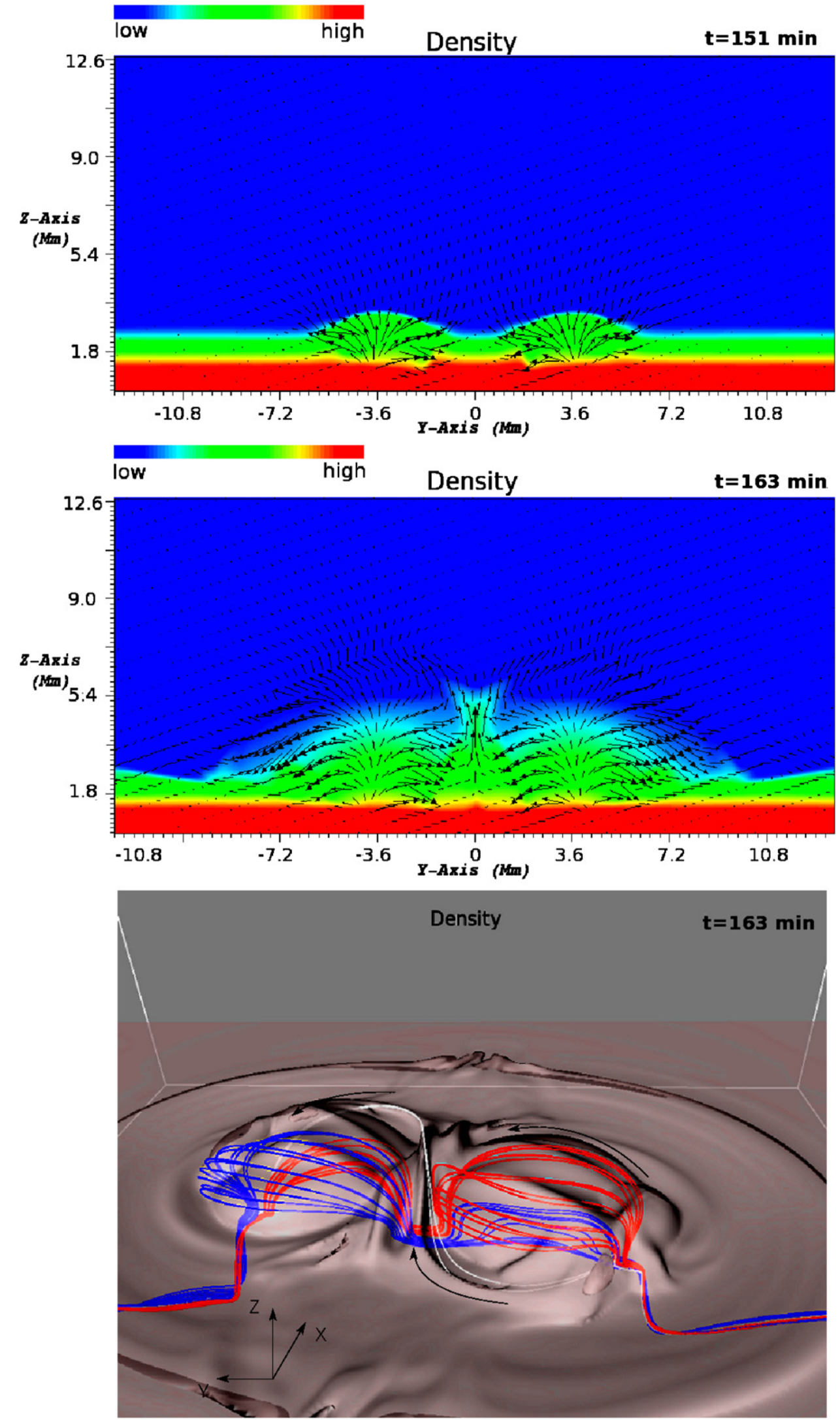

Figure 4. Top: density distribution at the vertical $y z$-midplane at $t=151$ minutes. Overplotted is the full velocity field vector. Middle: the same at $t=163$ minutes. Bottom: 3D isosurface of density at $t=163$ minutes. Blue and red fieldlines have been traced from the two magnetic lobes, while white fieldlines have been traced from the interface. The (black) arrows show the direction of the field along the fieldlines.

(A color version of this figure is available in the online journal.) 
field brings dense photospheric/chromospheric plasma into the upper atmosphere. Eventually, the heavy plasma drains from the summits of the expanding fieldlines of the lobes and is evidently collected at the dips of the $U$-shaped undulating fieldlines. Moreover, as the two lobes approach each other, background plasma is pushed toward the interface between the central polarities, where a thin and dense plasma layer is formed. There, the plasma is compressed, undergoing an apparent rising motion (middle panel) driven by the gas pressure gradient force. The associated vertical plasma flow is cool and dense, and it is physically different from the hot jets, which are eventually emitted from the interface due to reconnection.

The rising plasma is gradually oriented along the transverse direction, above the PIL between the central polarities of the EFR, where the total pressure is less. In the 3D space (bottom panel), the heavy plasma at the interface forms a cool loop with an arch-like configuration that extends from the photosphere into the low corona. The outermost fieldlines at the center of the initial tube ( $x=0 \mathrm{Mm}, y=0 \mathrm{Mm})$ are also displaced upward. These are the white fieldlines in the bottom panel of Figure 4 that overlie the dense loop. In fact, the rising material of the loop cannot break out through these fieldlines; thus, it remains confined within the EFR.

Eventually, a considerable amount of dense plasma has moved from the interface into the corona. This brings the oppositely directed fieldlines of the magnetic lobes closer together, and a strong current sheet builds up at the interface. Figure 5 (top panel) shows a 3D representation of the fieldline topology around the interface at $t \approx 169$ minutes. The current sheet is shown by the red isosurface of strong current magnitude $(|\mathbf{j}|=$ 0.25 ). Only the segment of the current sheet above $z \approx 2.1 \mathrm{Mm}$ is shown. The horizontal slice shows the distribution of $\mathrm{Bz}$ at the photosphere. The red (blue) fieldlines have been traced from the upper part (i.e., $(x, y, z)=(0, \pm 4.2,7.2) \mathrm{Mm})$ of the magnetic lobe at the front (far) side of the domain. These fieldlines have already undergone shearing as the two central polarities (i.e., $B_{z}$ in red and blue color at the center of Figure 5) move in opposite directions on the two sides of the PIL between the lobes.

At time $t \approx 168-172$ minutes, reconnection of the fieldlines occurs at $z \approx 2.5 \mathrm{Mm}$, along the vertical current sheet. This leads to the formation of two new magnetic flux systems. The first system consists of reconnected fieldlines that join the side polarities of the EFR directly from above (white fieldlines in Figure 5, top). These fieldlines have been traced from just above the current isosurface at the vertical $y z$-midplane. The upper segments of these fieldlines form, practically, untwisted magnetic loops in the corona, which in turn make an ambient field for the upcoming flux. The second system consists of strongly twisted fieldlines, which are lying underneath the vertical current sheet and extend along the PIL between the lobes, forming a post-emergence rope with a finite length. These are the yellow fieldlines shown in Figure 5 (top panel), which have been traced from the area just underneath the current isosurface. After they leave the central part of the postemergence flux rope, they establish links with both magnetic lobes. This is also apparent with the red fieldlines, the lower segments of which are involved in complex reconnection at the vicinity of their $U$-shaped dips, and as a result helically twisted fieldlines form, which they contribute significantly to the twist buildup of the post-emergence flux rope. The appearance of twist starts when the lower segments of the lobe fieldlines that reconnect undergo a continuous shearing and convergence. Note that this shearing is due to the oppositely directed motion of the central polarities of the EFR. Thus, although the initial emerging magnetic field is weakly twisted, reconnection of the sheared fieldlines at low atmospheric heights leads to the formation of a strongly twisted post-emergence flux rope above the axis of the original emerging flux tube.

The bottom panel in Figure 5 is a close-up of the postemergence flux rope at a later time ( $t=191$ minutes). The $3 \mathrm{D}$ topology of the fieldlines reveals the highly twisted nature of the magnetic field along the PIL. The post-emergence flux rope extends almost all along the neutral line. The fieldlines have been traced from the close neighborhood of the center of the post-emergence flux rope, at various positions along the $x$ - and $y$-direction (i.e., from $x=-1$ to $x=1$ and from $y=-1$ to $y=1$ ). It is shown that they join the post-emergence flux rope with the two lobes. At this stage of evolution, the central body of the post-emergence flux rope resides at the upper photosphere. As time goes on, the post-emergence rope rises slowly, but it does not emerge bodily into the corona. The central rope's segment is confined by the tension of the ambient fieldlines. Only the fieldlines at the ends of the post-emergence flux rope, which are closer to the two lobes, start to expand toward larger heights. This lateral expansion triggers reconnection with the ambient fieldlines, which is discussed in the next subsection.

\subsection{Heating and Jets}

The interaction between the magnetic lobes leads also to fast plasma ejection and increase of thermal energy via Joule dissipation. Figure 6 shows the two-dimensional (2D) distribution of temperature and density at the vertical $y z$-midplane at later times ( $t \geqslant 168$ minutes). Overplotted is the projected full velocity field vector. The fast ejection manifests itself in a pair of hot bi-directional jets that are emitted vertically from the reconnection site (top left panel). The upward jet can reach speeds of $\approx 70 \mathrm{~km} \mathrm{~s}^{-1}$ before it is diverted sideways along the reconnected fieldlines in the corona. This diverging motion is due to the effective confinement of the plasma by the overlying field. In fact, the jet is spatially divided into two distinct plasma flows that are running along the positive and negative $y$-direction (middle panel). The hot plasma is transported by the jets into the corona, heating up the apex of the two lobes. More precisely, it is displaced above the cold dense material of the two lobes, which has emerged into the corona after the initial adiabatic expansion of the field. The maximum temperature along the jets is approximately $1.5 \mathrm{mK}$. The density distribution (right panels, Figure 6) shows that the ejected plasma is also dense since it is literally coming from the reservoir of dense plasma, formed earlier at the interface. Thus, the dynamical interaction of the two lobes results in the formation of successive (cool and hot) dense coronal loops within the EFR.

Hot plasma from reconnection also flows down, from the vertical current sheet into the twisted magnetic flux system that formed earlier. This is clearly seen around $y=0 \mathrm{Mm}$, $z=0.36 \mathrm{Mm}$ in the top left panel in Figure 6. The area underneath the sheet adopts the configuration of a hot arcade, with a temperature of about $\approx 2 \mathrm{mK}$, and high (total) pressure. Gas pressure increases due to the plasma heating. Magnetic pressure is locally enhanced because new flux is added to the postemergence flux rope via reconnection. Initially, the size of the post-emergence flux rope does not change dramatically, since the outward expansion is balanced by the inward tension of the twisted fieldlines. Also, the post-emergence flux rope does not undergo a considerable horizontal expansion because it is located between the two lobes that exert magnetic pressure forces 

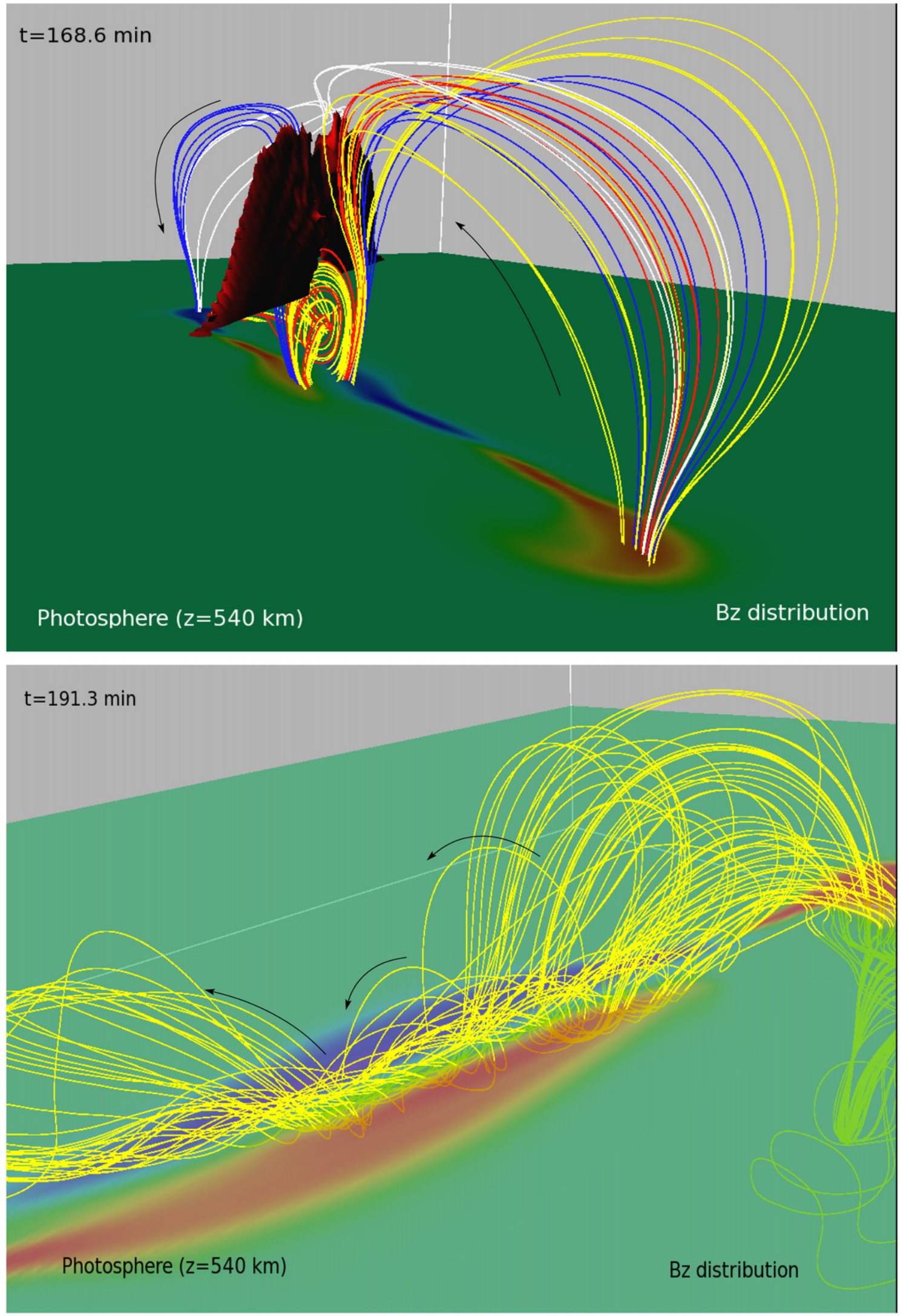

Figure 5. Top: three-dimensional topology of fieldlines at $t=168.6$ minutes. The isosurface shows high magnitude of current. The horizontal plane shows the $B_{z}$ distribution: red (blue) is positive (negative). The visualized range of $B_{z}$ is $(-300,300)$ G. Bottom: fieldlines showing the twisted nature of a newly formed (post-emergence) flux rope at the photosphere. The black arrows indicate the direction of the field.

(A color version of this figure is available in the online journal.) 

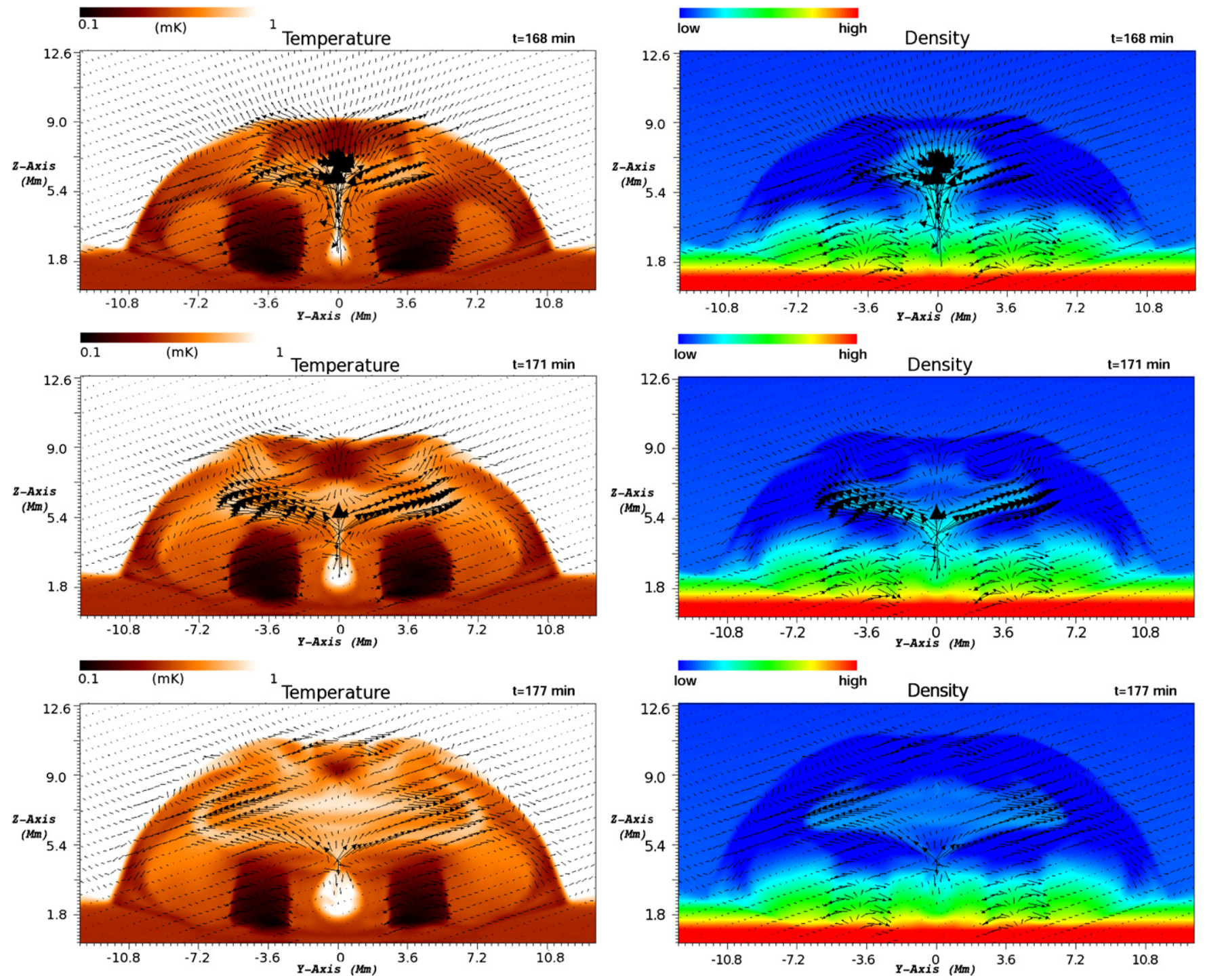

Figure 6. Left: temperature distribution at the $x=0 \mathrm{Mm}$ slice. Arrows show the projected velocity field onto the plane. Right: the same for density.

(A color version of this figure is available in the online journal.)

toward the post-emergence flux rope. Due to the above, the post-emergence flux rope encounters more vertical stretching and vertical expansion than profound horizontal spreading.

The local pressure enhancement underneath the current sheet induces a bi-directional outflow that is oriented parallel to the PIL between the lobes. Figure 7 (left panel) is a closeup, showing the gas pressure distribution as 3D transparent isosurface above $z \approx 2 \mathrm{Mm}$. The arch-shaped structure at the center of the EFR corresponds to the high-pressure regime, with a value of about 1-2 orders of magnitude higher than the surrounding plasma pressure at the same height. The fieldline topology confirms the good spatial correlation between the highly twisted structure and the region of high plasma pressure. Overplotted is the full velocity field vector, colored by the magnitude of the horizontal velocity (red (blue) represents high (low) velocity). The expansion of the lobes is apparent through the fan-like shape of the fieldlines sideways from the center of the EFR. At the ends of the high-pressure regime, there is the horizontal bi-directional outflow with a velocity that reaches values up to $6-7 \mathrm{~km} \mathrm{~s}^{-1}$. As a result of this outflow, the density is expelled from the ends of the high-pressure regime toward the outskirts of the EFR, where pressure is low. The expulsion of the heavy plasma is mainly horizontal in nature and starts at $t>175$ minutes. Gradually, it leads to a density deficit within the post-emergence flux rope. This process makes the post-emergence flux rope less dense and more buoyant; consequently, after $t=191$ minutes, it rises slowly toward the upper atmosphere.

The ends of the post-emergence flux rope, which are located closer to the lobes, encounter also vertical stretching and expansion as they rise. Eventually they come into contact with the overlying fieldlines and reconnect. The topology of the fieldlines around such a reconnection site (at $x=0 \mathrm{Mm}$, $y=3.6 \mathrm{Mm}, z=6.3 \mathrm{Mm})$ is shown in the right panel of Figure 7. The overlying fieldlines (green) are previously reconnected fieldlines that join, directly from above, the two main polarities of the EFR and point along the $y$-direction. The fieldlines that belong to the post-emergence flux rope (yellow) have more azimuthal nature and join the two side polarities at the photosphere. The newly reconnected fieldlines (blue and red) establish links between the twisted flux structure from the lower atmosphere and the coronal loops of the ambient field. 


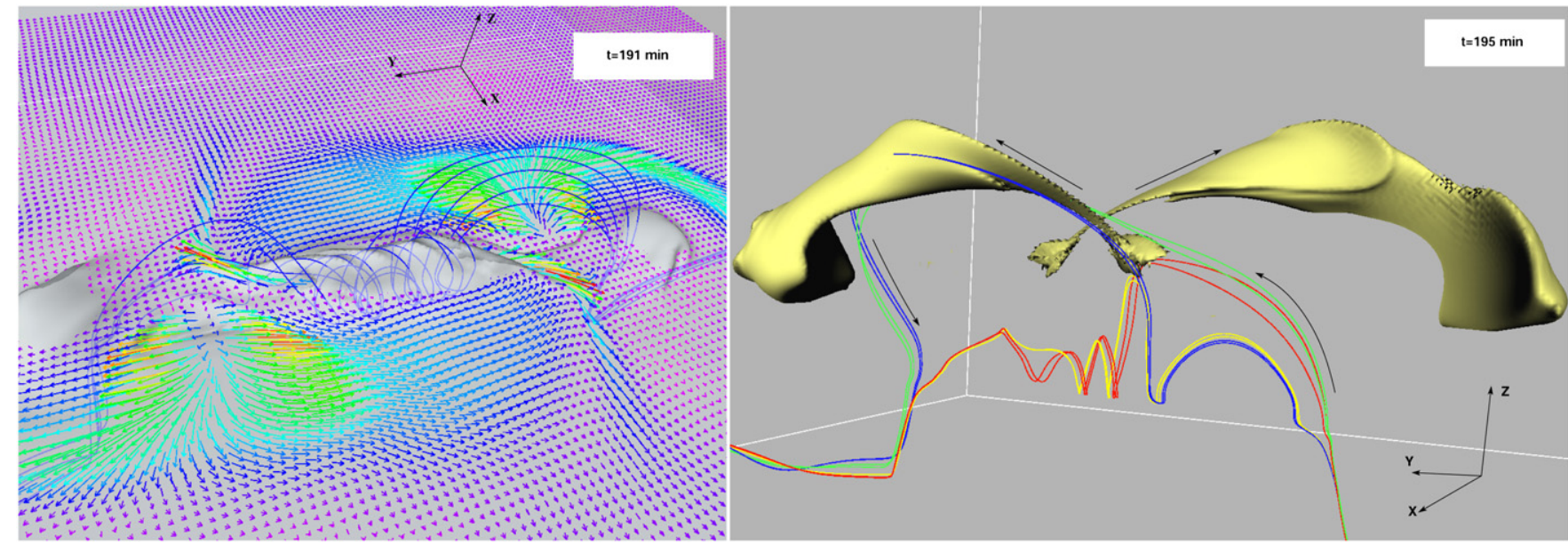

Figure 7. Left: visualization of the high-pressure regime (gray transparent isosurface) at the center of the EFR. The arrows show the full velocity field vector at $z=2.1 \mathrm{Mm}$. The magnetic fieldlines resemble the expansion of the magnetic lobes and the twisted nature of the flux tube at the middle of the EFR. Right: the reconnection jets (yellow isosurface) with a speed of about $20 \mathrm{~km} \mathrm{~s}^{-1}$. The fieldlines show the reconnection between the emerging and the overlying fieldlines. Arrows indicate the direction of the field along the fieldlines.

(A color version of this figure is available in the online journal.)

Reconnection occurs, almost symmetrically, at both ends of the post-emergence flux rope. The magnitude of the velocity field (overplotted yellow isosurface) shows the emission of a pair of jets, which emanate from the reconnection sites. The 3D structure of the jets reveals that they follow a curved path as they are running along the reconnected fieldlines. The two jets run in opposite directions (toward the $\pm y$-direction), moving toward the main polarities of the EFR. Their emission is accompanied by marked heating $(1-2 \mathrm{mK})$ of the plasma. The hot emitted material is transported from the corona to the lower atmosphere, giving rise to an apparent heating of the plasma at successively lower heights.

To estimate the magnitude of the vertical flows due to the interaction of the two lobes, over the whole time evolution of the system we calculate the maximum value of $v_{z}$ from the photosphere and above. The emergence of the first magnetic flux elements at the photosphere proceeds with velocities of about $2-3 \mathrm{~km} \mathrm{~s}^{-1}$. Thus, flows with higher speeds might account for motions due to interaction (e.g., reconnection) between the magnetic lobes and not due to the direct emergence of the magnetic flux from the sub-photospheric domain. The top left panel in Figure 8 shows the time evolution of the maximum value of the vertical velocity field component. The top speed of $v_{z}=70 \mathrm{~km} \mathrm{~s}^{-1}$ at $t \approx 168$ minutes corresponds to the first reconnection jet, which was emitted from the interface between the lobes. More jets, albeit with lower speeds, occur at later times. For instance, the pronounced plasma motion at $t=210$ minutes matches the ejection of the horizontal jets due to reconnection between the rising and the overlying magnetic fieldlines. After $t \approx 260$ minutes, the vertical flows obey a less episodic behavior, decreasing to values of about $v_{z}=4-5 \mathrm{~km} \mathrm{~s}^{-1}$ at the end of the simulation. The vast majority of these late (subsonic and sub-Alfvénic) flows occur in the upper atmosphere due to compression or weak reconnection of the tangled fieldlines within the EFR.

The dynamic evolution of the emerging flux system leads also to the transfer of dense and hot plasma into the upper atmosphere. This has been already revealed in the 2D cuts in Figure 6. To get additional quantitative estimates of how much hot/dense plasma is rising into the atmosphere, we calculate (top right and bottom panels in Figure 8) the time evolution of the (dimensionless) ratio of the maximum temperature and maximum density at a certain atmospheric height $(z)$, over the corresponding value of the initial background plasma at this height. For instance, the top right panel shows this ratio (for temperature (blue) and for density (red)) at the middle of the transition region. The rapid increase of temperature at $t \geqslant 150$ minutes corresponds to the hot plasma emission due to reconnection between the two lobes. A comparison with the evolution of $v_{z}$ reveals that the plasma is heated during the period ( $t=144-274$ minutes) of pronounced vertical (reconnection) jets. After this period, the plasma temperature returns to its nominal value of the unperturbed atmosphere. Note that the interaction of the lobes feeds the transition region with dense plasma and that there is a good correlation between the onset of hot plasma emission and the deposition of dense material at transition region heights. It is remarkable that even after the high-velocity emission ceases, the dense material still remains at these heights, although it undergoes a small decrease mainly due to gravitational draining.

Now we evaluate the plasma response at coronal heights. The bottom panels in Figure 8 show the temperature and density evolution at $z=5.4 \mathrm{Mm}, z=6.3 \mathrm{Mm}$, and $z=7.2 \mathrm{Mm}$. Initially (up to $t=164$ minutes), corona is unperturbed. Eventually, the emergence of the magnetic field brings cool and dense material from the lower atmosphere, at times between $t=$ 164 minutes and $t=188$ minutes. Then, reconnection (which occurs either at lower atmospheric heights or locally within the corona) results in the transport of hot and dense plasma to the upper atmosphere. Note that the plasma temperature at these coronal heights can increase by nearly a factor of three, which is much less than the relative temperature enhancement in the transition region. This implies that (1) the reconnecting plasma that is produced at lower atmospheric heights is hotter and remains there (e.g., see middle left panel in Figure 6) and/or (2) reconnection at the corona is not so effective in producing equally hot plasma. In any case, the distribution of the temperature (bottom left panel) reveals that there is a similar temporal evolution at all coronal heights. After the temperature enhancement, there is a gradual decrease that eventually brings the plasma close to its original value (that of the unperturbed corona). This is not the case for the density variation. After 

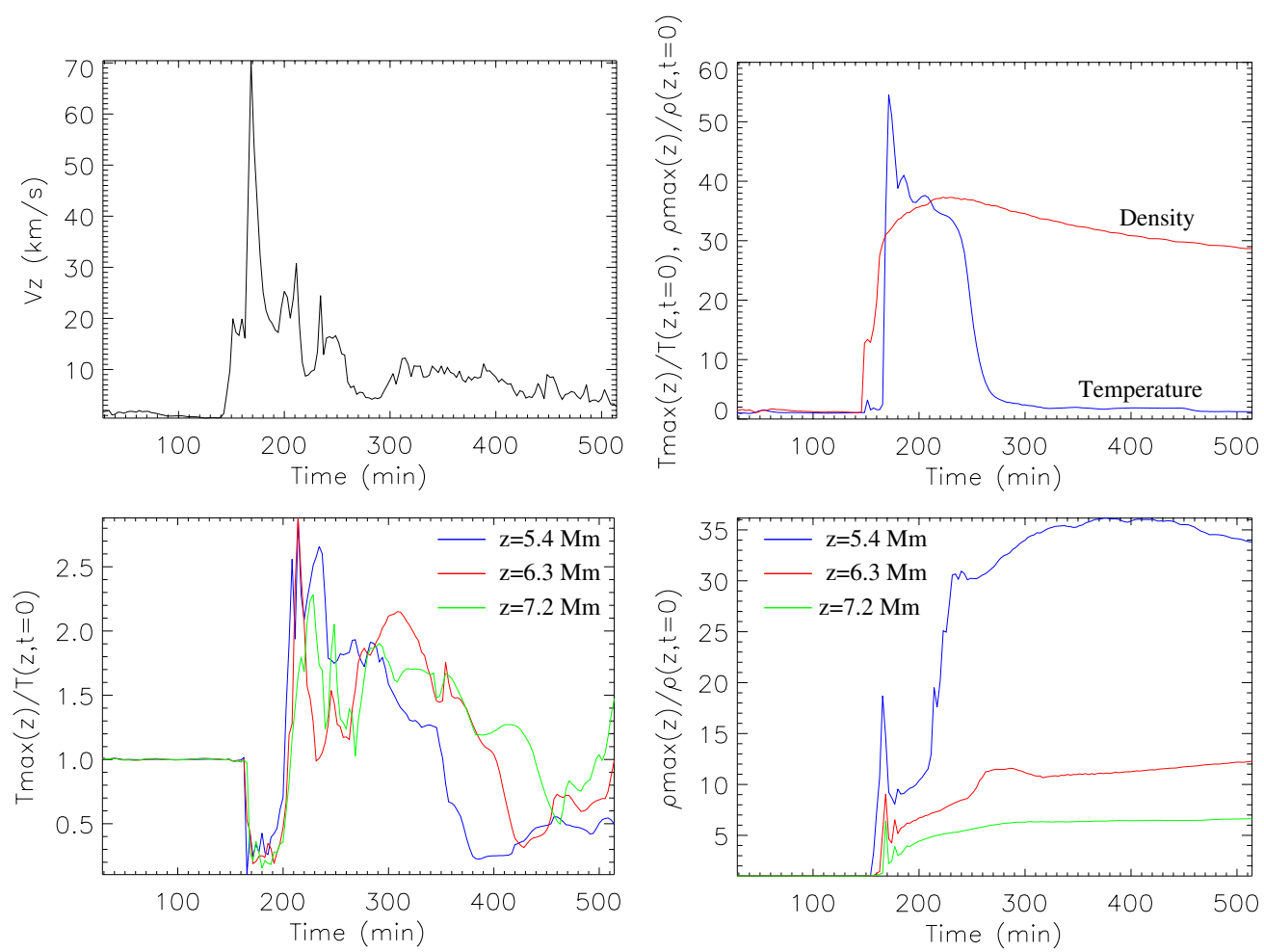

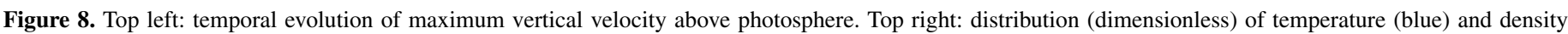

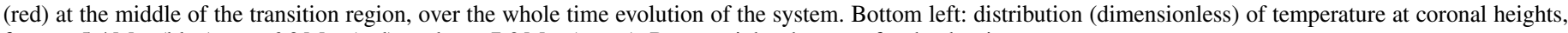
for $z=5.4 \mathrm{Mm}$ (blue), $z=6.3 \mathrm{Mm}$ (red), and $z=7.2 \mathrm{Mm}$ (green). Bottom right: the same for the density.

(A color version of this figure is available in the online journal.)

$t \approx 188$ minutes, plasma density increases until it saturates at a level that is higher than that of the unperturbed background atmosphere. Most of the dense plasma that enters the corona remains at low heights. Nevertheless, the above study reveals that this EFR is capable of sustaining hot and dense plasma, which is distributed along loops of magnetic fieldlines, at all atmospheric heights.

\section{FLUX TRANSFER}

During the dynamical evolution of the emerging field, magnetic flux is transferred from the solar interior to the surface and toward the outer solar atmosphere. First, we calculate the Poynting flux at the photosphere. Following Kusano et al. (2002) and Magara \& Longcope (2003), we divide the Poynting flux into two terms: the emergence term

$$
F_{\text {emergence }}=\frac{1}{4 \pi} \int_{z_{0}}\left(B_{x}^{2}+B_{y}^{2}\right) v_{z} d x d y,
$$

which represents the direct emergence of the magnetic field, and the shear term, which implies the work done by the horizontal motions:

$$
F_{\text {shear }}=-\frac{1}{4 \pi} \int_{z_{0}}\left(B_{x} v_{x}+B_{y} v_{y}\right) B_{z} d x d y \text {. }
$$

The top panel in Figure 9 shows the time evolution of the two terms at three different heights within the photosphere: $z_{0}=0.18 \mathrm{Mm}$ (black), $z_{0}=0.68 \mathrm{Mm}$ (blue), and $z_{0}=0.9 \mathrm{Mm}$ (red). At all heights, the flux system is dominated by the emergence term at the initial phase and by shear at a later stage. Both terms decrease with height: they are more pronounced at the base of the photosphere than in the middle or at the top of the photosphere. At each height, the temporal evolution shows that emergence and shear reach their maximum value first and then they decrease until the end of the experiment. The above results are partially consistent with the observational work by Liu \& Schuck (2012), who calculated the magnetic energy (Poynting) flux in ARs. They found that the emergence and shear-energy fluxes evolve consistently in phase during the entire flux emergence course. This is similar to the evolution found in our model. One difference is that in our simulations, there is a very short impulsive injection of energy from the emergence term at the beginning of flux emergence. This is found also in other numerical experiments (e.g., Magara \& Longcope 2003). The observations do not show this impulsive behavior. However, it is worthwhile mentioning that our experiments show that this impulsive phase disappears at larger heights within the photosphere (e.g., at $z_{0}=0.68 \mathrm{Mm}$ and $z_{0}=0.9 \mathrm{Mm}$ ), which is more consistent with the observational measurements. An interesting result found by Liu \& Schuck (2012) is that the energy flux in ARs is mainly contributed by the emergence term during flux emergence. This is partially consistent with our simulations, which show that the emergence term is important only at the beginning while the shear term becomes dominant for the rest of the system's evolution. This is probably due to the fact that there is no continuous flux emergence (that could be driven by fully developed convection) in our experiments.

To study how much flux is transferred to the solar atmosphere, we calculate the axial flux

$$
\Phi_{y}(t)=\int_{-25}^{25} \int_{z \geqslant z_{0}}^{45} B_{y} d x d z
$$



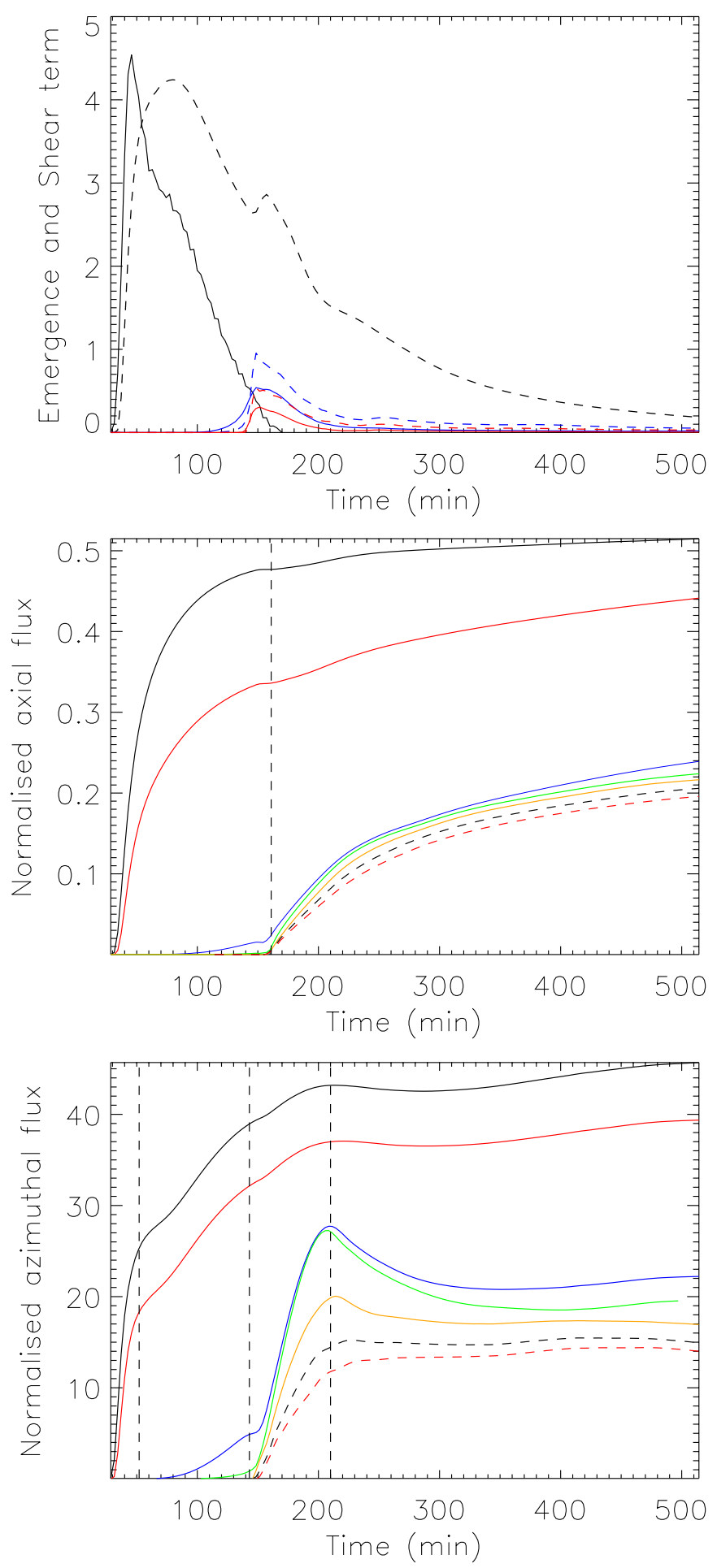

Figure 9. Top: temporal evolution of $F_{\text {emergence }}$ (solid) and $F_{\text {shear }}$ (dashed) at $z_{0}=0.18 \mathrm{Mm}$ (black), $z_{0}=0.68 \mathrm{Mm}$ (blue), and $z_{0}=0.9 \mathrm{Mm}$ (red). Middle: temporal evolution of the normalized axial flux at $z=0 \mathrm{Mm}$ (black), $z=0.18 \mathrm{Mm}$ (red), $z=0.68 \mathrm{Mm}$ (blue), $z=0.9 \mathrm{Mm}$ (green), $z=1.8 \mathrm{Mm}$ (orange), $z=2.6 \mathrm{Mm}$ (dotted black), and $z=3.4 \mathrm{Mm}$ (dotted red). Bottom: the same for the normalized azimuthal flux. The vertical dashed lines are placed at times $t=51$ minutes, $t=144$ minutes, and $t=210$ minutes, denoting the changes in the evolution of the flux.

(A color version of this figure is available in the online journal.)

at the $x z$-midplane. The middle panel in Figure 9 shows the time evolution of $\Phi_{y}(t)$ above certain heights, normalized by the initial axial flux of the emerging tube. It is shown that, at the end of the simulation, a considerable amount of flux (above
$50 \%$ ) has crossed the surface and has been transferred above the base of the photosphere. However, most of this flux remains within a few pressure scale heights, and only $\approx 20 \%$ emerges above $z_{0}=3.4 \mathrm{Mm}$.

At $z_{0}=0 \mathrm{Mm}, z_{0}=0.18 \mathrm{Mm}$ the initial rapid increase of $\Phi_{y}(t)$ is due to direct emergence of the field. In general, for all heights, there is a good correlation between the period during which the emergence term ( $F_{\text {emergence }}$ in top panel) increases and the first stage of enhancement of $\Phi_{y}(t)$. At $t \approx 154-161$ minutes, a marked increase of $\Phi_{y}(t)$ starts to occur for heights $z_{0} \geqslant 0.68 \mathrm{Mm}$. This is mainly due to the shearing of the field as this is the dominant motion during this stage of evolution (see also top panel). The shearing of the fieldlines that pass through the magnetic lobes and their subsequent reconnection increase the axial field component at the $x$-midplane; therefore, the axial flux within the EFR increases too.

Note that there is a moderate increase of $\Phi_{y}(t)$ at even lower heights (e.g., $z_{0}=0 \mathrm{Mm}, z_{0}=0.18 \mathrm{Mm}$ ) around $t=$ 161 minutes. This correlates well with the increase in the shear term. The temporal increase of $F_{\text {shear }}$ is because the arch-like reconnected fieldlines that intersect the low photosphere have a vertical-field $\left(B_{z}\right)$ component, forming a new weak bipolar region between the two main polarities of the EFR. Eventually, these reconnected fieldlines encounter shearing, following the general evolution of the system. The above processes cause the second boost in the shear term and the slow increase of $\Phi_{y}(t)$ after $t=154$ minutes.

Although the initial twist of the emerging flux tube is weak, there is a non-negligible amount of azimuthal flux transferred into the photosphere and above. In previous studies (e.g., Murray et al. 2006), it has been shown that the azimuthal field component increases already while the tube rises within the sub-photospheric layer. Also, the emergence is followed by expansion of the field that supports the increase of the azimuthal field. The bottom panel in Figure 9 shows the time evolution of the signed azimuthal flux

$$
\Phi_{x}(t)=\int_{-25}^{25} \int_{z \geqslant z_{0}}^{45} B_{x} d y d z
$$

at the vertical $y z$-midplane (normalized by the initial unsigned $\Phi_{x}$ at $t=0$ minutes). Close to the base of the photosphere (i.e., $z=0 \mathrm{Mm}, z=0.18 \mathrm{Mm}$ ), the initial increase of $\Phi_{x}(t)$ (up to $t=51$ minutes) is due to the direct emergence of the field. The latter has been illustrated in the top panel by the emergence term. After $t=51$ minutes, the gradual increase of $\Phi_{x}(t)$ is due to the additional compression of the magnetic field after the squeezing and the rising motion of the dense plasma between the lobes. Indeed, the bottom panel in Figure 4 has revealed the strongly azimuthal nature of the uppermost fieldlines at the center of the EFR after the compression of the field. The two-loop emergence and the general expansion of the field contribute also to the increase of $\Phi_{x}(t)$. Finally, the increase between $t=144$ minutes and $t=210$ minutes is due to the formation of the twisted flux tube via reconnection of the lobes. The twisted fieldlines of the post-emergence flux rope have a strong azimuthal component resulting in an increase of the corresponding flux.

This is more clearly evident for heights $z \geqslant 0.68 \mathrm{Mm}$. The horizontal layer at $z_{0}=0.68 \mathrm{Mm}$ intersects the post-emergence flux rope just below its center, when it is initially formed. Thus, the rapid increase of $\Phi_{x}(t)$ at $t=144$ minutes represents 
the azimuthal nature of the fieldlines at the upper part of the post-emergence flux rope. As the whole rope rises above this height, the signed $B_{x}$ contribution to the flux by the twisted rope is reduced. Eventually, $\Phi_{x}(t)$ approaches saturation or even gradual decrease when the twisted fieldlines of the rising flux tube start to reconnect with the surrounding field, losing their strongly azimuthal nature. The temporal evolution of $\Phi_{x}(t)$ is similar at larger heights (e.g., within the transition region $\left(z_{0}=\right.$ $2.6 \mathrm{Mm})$ and corona $\left(z_{0}=3.4 \mathrm{Mm}\right)$ ). In general, the azimuthal flux within the photosphere reaches a saturation level after $t=210$ minutes. However, even at larger heights the azimuthal flux does not change dramatically after this time. It is worthwhile mentioning that at the end of the evolution, the azimuthal flux above the photosphere is 15 times larger, and within the transition region and low corona it is $\approx 14$ and $\approx 13$ times larger, respectively (on average), than that of the initial flux tube.

\section{CONCLUSIONS AND DISCUSSION}

In this paper we studied the emergence of a weakly twisted magnetic flux tube through the highly stratified atmosphere of the Sun. The main results of our work are briefly described as follows.

1. The weak twist results in the emergence of two magnetic lobes. Thus, an undulating system forms naturally as a result of a weakly twisted field that emerges from the solar interior.

2. The interaction of the lobes leads to the ejection of (1) cool and dense outflows from the interface, driven by local enhancement of plasma pressure, and (2) hot jets due to reconnection between the fieldlines of the lobes, driven by the Lorentz force.

3. Shearing and reconnection of the fieldlines at low atmospheric heights lead to the formation of a strongly twisted flux tube that does not undergo an ejective full eruption. It is confined by the overlying field that is formed by the expansion and the reconnection between the fieldlines of the magnetic lobes.

4. Eventually, the post-emergence flux rope (mainly its flanks close to the main polarities of the system) expand into the atmosphere and start reconnecting with the ambient field. This process (self-induced reconnection) triggers the emission of hot lateral jets within the EFR.

5. Although the initial sub-photospheric system is a low plasma- $\beta$ and with weak twist, a considerable amount of (axial and azimuthal) flux is transported above the solar surface.

Under the specific initial conditions in our experiment, the above results strongly indicate that strong twist is not necessary for the magnetic field to emerge above the photosphere. There is a partial (two-loop) emergence of the weakly twisted magnetic field into the corona leading to the formation of an undulating EFR. The topology of the emerging field is reminiscent of the "sea serpent" configuration, which has been observed in EFRs (e.g., Watanabe et al. 2008; Centeno 2012; Valori et al. 2012). This is probably the result of interaction between granular convection and magnetic fields, resulting in the appearance of small-scale magnetic bipolar structures on granular scales (e.g., Cheung et al. 2008; Tortosa-Andreu \& Moreno-Insertis 2009; Bushby \& Archontis 2012). On the other hand, it has been found that undulations in "sea serpent" fields have a wavelength that is comparable to the most unstable wavelength of the Parker instability (i.e., $\approx 4 \mathrm{Mm}$ at the photosphere; i.e., Pariat et al. 2004; Otsuji et al. 2011). In addition, numerical simulations of long emerging flux sheets (e.g., Isobe et al. 2007) have demonstrated the gradual appearance of a large number of interconnecting $\Omega$ loops that originate from the sheet as a result of the Parker instability. Based on this result, one would expect that a considerable increase (e.g., by a factor of four) of the length $\lambda$ of the buoyant part of the initial tube in the present experiments would result in more undulations. However, this is not the case: in experiments that we have performed (not shown in the present study), we found that the field becomes buoyant again in two places close to the main polarities of the flux system. The two-loop emergence occurs naturally when the criterion for the magnetic buoyancy instability of the photospheric field is satisfied (see Section 3.2).

In addition to this experiment, we performed numerical simulations (not shown here) to study the rising motion of a non-twisted magnetic flux tube through the solar atmosphere. We found that the magnetic field is still able to rise and expand into the corona, although the local properties of the side effects and the temporal/spatial evolution of the system are different. This reinforces our conclusion that in these highly idealized experiments, twist is not a key requirement for the emergence of the field. Dynamics is mainly driven by the magnetic field; thus, it is the values of the field strength and the plasma- $\beta$ of the rising field that are the crucial parameters for determining the actual flux emergence.

The interaction of the magnetic lobes leads to the emission of flows that are driven either by reconnection (hot jets) or by the gradient of the plasma pressure (cool and dense outflows) at the interface between the magnetic lobes. In both cases, the associated plasma emission is confined within the EFR, forming a system of hot and cool loops that arch over the emerging field. Therefore, this mechanism should be considered as a possible driver for the formation of coronal loops of different temperatures that have repeatedly been observed to coexist in ARs and arch-filament systems (e.g., Isobe et al. 2005). The cool outflows, which are emitted before the reconnection jets, might account for $\mathrm{H} \alpha$ surges in EFRs (e.g., Canfield et al. 1996; Liu \& Kurokawa 2004; Jiang et al. 2007). Similar flows have also been found to form in 2D and 3D numerical experiments of emerging fields with an undulating configuration (e.g., Isobe et al. 2007; Archontis \& Hood 2009). They form at the valleys between interacting magnetic fields due to a pressure gradient force. A similar mechanism has been used to explain the emission of cool outflows associated with the emergence of new magnetic flux at the outskirts of an AR (e.g., Harra et al. 2012). The cool emission originates from the area where the interacting magnetic fields experience more compression than reconnection. On the other hand, the hot reconnection jets that are produced in the present simulations could be observed as X-ray jets (e.g., Shibata et al. 1992). In previous studies, similar jets were produced as a result of reconnection between emerging and preexisting magnetic fields (e.g., Archontis et al. 2010; Archontis \& Hood 2013).

Another by-product of the interaction of the magnetic lobes is the formation of a highly twisted post-emergence flux rope. This is a striking result considering the fact that the emerging subphotospheric field is only weakly twisted. The post-emergence flux rope is formed due to shearing and reconnection of the fieldlines as they are brought into contact with each other. It is formed along the PIL between the lobes and rises slowly without being able to erupt in an ejective manner, toward the outer solar atmosphere. Our experiment clearly demonstrates that the existence of a twisted field above a PIL within an EFR 
or an AR does not necessarily imply that a twisted magnetic field has emerged from the solar interior.

The formation and eruption of a post-emergence flux rope, after the emergence of a twisted magnetic field, have been investigated in various numerical experiments in the past (e.g., Manchester et al. 2004; MacTaggart \& Hood 2009b; Archontis \& Török 2008; Archontis \& Hood 2010, 2012). A common result in these experiments is the formation of a single bipolar structure at the photosphere and the formation of a postemergence rope due to the shearing and internal reconnection of the fieldlines along the PIL between the two main polarities of the bipole. Therefore, the topology of the field prior to the formation of the post-emergence flux rope is quite different compared to the present simulations where this new flux rope is formed due to reconnection between two magnetic lobes. A key result in some of the previous studies (e.g., Archontis \& Török 2008; Archontis \& Hood 2010, 2012) was the ejective eruption of the post-emergence flux rope when sufficient external reconnection occurred between the emerging and an ambient magnetic field. However, the eruption was confined when the emergence was taking place in a fieldfree atmosphere. In the latter case, the post-emergence flux rope could rise up into the corona where it reached a quasistatic equilibrium when the downward tension of the uppermost expanding fieldlines (i.e., envelope field) was balanced by the Lorentz force that drove the eruption of the rope. Therefore, the confinement of a post-emergence flux rope has been shown in previous experiments, but it occurred over a different timescale. More precisely, the whole evolution (from the beginning of the sub-photospheric emergence until the eruption) occurred on a timescale that was very short $(\approx 1 \mathrm{hr})$ compared to the observations of eruptions in EFRs or ARs. However, in most observational examples, there is a considerable delay between the time at which the photospheric magnetic flux reaches its peak and the time at which the AR undergoes an eruptive activity. This is more consistent with the results of the present simulations. The emission of a hot reconnection jet and the formation of the post-emergence flux rope occur at $\approx 2.5-3 \mathrm{hr}$ after the initial emergence. The central segment of the post-emergence flux rope does not experience an ejective eruption: it remains confined at low atmospheric heights throughout the evolution of the system (for about 7-8 hr). A preliminary comparison with the previous experiments (e.g., Archontis \& Török 2008; Archontis \& Hood 2010,2012 ) indicates that it is probably (the combination of) the low value of twist and the smaller field strength of the emerging flux tube $(2.8 \mathrm{kG}$ compared to the most common value of $6.5 \mathrm{kG}$ in previous experiments) that result in a more reasonable temporal and spatial evolution of the system.

To study the effect of the numerical resolution on the properties of the jets, we have performed a numerical experiment with lower resolution (by a factor of 1.6 compared to the present model), and we have found that the velocity of the reconnection jet at $t=168$ minutes is smaller $\left(v \approx 25-30 \mathrm{~km} \mathrm{~s}^{-1}\right)$. The maximum heating of the plasma during the emission of the jet in the low-resolution case is only 1.3 times stronger. On the other hand, we conjecture that for higher resolution than that of the present model, smaller scales will be resolved, but the general evolution of the system will not change dramatically. Other factors that affect the properties (velocity, plasma heating) of the jets are the inclusion of (a) extra energy sources or sinks in the energy budget of the system (e.g., heat conduction, radiative transfer, etc.) and (b) the state of ionization of the plasma. The above options ((a) and (b)) have not been considered in the present experiments; therefore, the exact values of the temperature and the speed of the jets may be somewhat different. For instance, here we have considered a weakly ionized plasma; thus, the temperature of the jets in the corona is likely to be overestimated by at most a factor of two. Despite the differences in the exact values of plasma heating and velocity, we believe that we will still get (X-ray and EUV) jets in a similar manner (i.e., the mechanism of the formation of the jets is based on the dynamics and not on the thermodynamical properties of the system) to the present model.

A common discussion topic in numerical simulations is the effect of explicit resistivity on the evolution of the system. Our previous numerical experiments in a similar model (i.e., interaction between two emerging flux tubes by Archontis et al. 2007) have shown that (1) the formation of structures (e.g., plasmoids) within the interface current is a robust result that does not strongly depend on the resistivity model once there is enough resolution to resolve these scales and (2) a decrease of the uniform resistivity (e.g., from $\eta=10^{-2}$ to $\eta=10^{-3}$ ) results in the increase of the maximum reconnection rate within the interface by a small factor (between 1.2 and 1.3). In addition, a non-uniform resistivity model (i.e., the resistivity is locally enhanced when the current density exceeds a certain threshold) produces similar results with the uniform low-resistivity $\left(\eta=10^{-3}\right)$ case. Therefore, we conjecture that using the present resistivity model with $\eta=10^{-3}$, we do not overestimate the Joule heating that is produced during the emission of the jets.

The dynamic evolution of the post-emergence flux rope in association with the two-loop emergence has not been investigated in this work. It is possible that there is a transfer of helicity (self and mutual) between the post-emergence flux rope and the magnetic lobes. The energy budget and the possible helicity transfer into the corona, together with a full parametric study of weakly twisted and non-twisted emerging fields (that is required to explore, e.g., the properties of the produced flows/ jets within the EFR and the rising motion of the post-emergence flux rope), will be presented in future work.

The simulations were performed on the STFC and SRIF funded UKMHD cluster, at the University of St Andrews. K.T. and V.A. acknowledge EU support (IEF-272549 grant). V.A. and A.W.H. are grateful for in-depth discussions during the ISSI workshops: "Magnetic flux emergence in the solar atmosphere" and "Understanding solar jets" in Bern.

\section{REFERENCES}

Abbett, W. P., Fisher, G. H., \& Fan, Y. 2000, ApJ, 540, 548

Acheson, D. J. 1979, SoPh, 62, 23

Arber, T., Longbottom, A., Gerrard, C., \& Milne, A. 2001, JCoPh, 171, 151

Arber, T. D., Haynes, M., \& Leake, J. E. 2007, ApJ, 666, 541

Archontis, V., \& Hood, A. W. 2009, A\&A, 508, 1469

Archontis, V., \& Hood, A. W. 2010, A\&A, 514, A56

Archontis, V., \& Hood, A. W. 2012, A\&A, 537, A62

Archontis, V., \& Hood, A. W. 2013, ApJL, 769, L21

Archontis, V., Hood, A. W., \& Brady, C. 2007, A\&A, 466, 367

Archontis, V., Hood, A. W., Savcheva, A., Golub, L., \& Deluca, E. 2009, ApJ, 691,1276

Archontis, V., Moreno-Insertis, F., Galsgaard, K., \& Hood, A. W. 2005, ApJ, 635,1299

Archontis, V., Moreno-Insertis, F., Galsgaard, K., Hood, A., \& O'Shea, E. 2004, A\&A, 426, 1047

Archontis, V., \& Török, T. 2008, A\&A, 492, L35

Archontis, V., Tsinganos, K., \& Gontikakis, C. 2010, A\&A, 512, L2

Baumann, G., \& Nordlund, Å. 2012, ApJL, 759, L9

Bushby, P. J., \& Archontis, V. 2012, A\&A, 545, A107 
Canfield, R. C., Reardon, K. P., Leka, K. D., et al. 1996, ApJ, 464, 1016

Canou, A., \& Amari, T. 2010, ApJ, 715, 1566

Centeno, R. 2012, ApJ, 759, 72

Centeno, R., Socas-Navarro, H., Lites, B., et al. 2007, ApJL, 666, L137

Cheung, M. C. M., Schüssler, M., \& Moreno-Insertis, F. 2007, A\&A, 467, 703

Cheung, M. C. M., Schüssler, M., Tarbell, T. D., \& Title, A. M. 2008, ApJ, 687, 1373

Fan, Y. 2001, ApJL, 554, L111

Fan, Y., Abbett, W. P., \& Fisher, G. H. 2003, ApJ, 582, 1206

Gibson, S. E., \& Fan, Y. 2006, ApJL, 637, L65

Gontikakis, C., Archontis, V., \& Tsinganos, K. 2009, A\&A, 506, L45

Harra, L. K., Archontis, V., Pedram, E., et al. 2012, SoPh, 278, 47

Isobe, H., Miyagoshi, T., Shibata, K., \& Yokoyama, T. 2005, Natur, 434, 478

Isobe, H., Tripathi, D., \& Archontis, V. 2007, ApJL, 657, L53

Jiang, Y. C., Chen, H. D., Li, K. J., Shen, Y. D., \& Yang, L. H. 2007, A\&A, 469,331

Kusano, K., Maeshiro, T., Yokoyama, T., \& Sakurai, T. 2002, ApJ, 577, 501

Leka, K. D., Canfield, R. C., McClymont, A. N., \& van Driel-Gesztelyi, L. 1996, ApJ, 462, 547

Liggett, M., \& Zirin, H. 1985, SoPh, 97, 51

Lites, B. W., Kubo, M., Berger, T., et al. 2010, ApJ, 718, 474

Liu, Y., \& Kurokawa, H. 2004, ApJ, 610, 1136

Liu, Y., \& Schuck, P. W. 2012, ApJ, 761, 105

López Fuentes, M. C., Demoulin, P., Mandrini, C. H., \& van Driel-Gesztelyi, L. 2000, ApJ, 544, 540

Luoni, M. L., Démoulin, P., Mandrini, C. H., \& van Driel-Gesztelyi, L. 2011, SoPh, 270, 45
MacTaggart, D., \& Hood, A. W. 2009a, A\&A, 508, 445

MacTaggart, D., \& Hood, A. W. 2009b, A\&A, 507, 995

MacTaggart, D., \& Hood, A. W. 2010, ApJL, 716, L219

Magara, T., \& Longcope, D. W. 2003, ApJ, 586, 630

Manchester, W., IV, Gombosi, T., DeZeeuw, D., \& Fan, Y. 2004, ApJ, 610,588

Moreno-Insertis, F., \& Emonet, T. 1996, ApJL, 472, L53

Moreno-Insertis, F., Galsgaard, K., \& Ugarte-Urra, I. 2008, ApJL, 673, L211

Murray, M. J., Hood, A. W., Moreno-Insertis, F., Galsgaard, K., \& Archontis, V. 2006, A\&A, 460, 909

Okamoto, T. J., Tsuneta, S., Lites, B. W., et al. 2008, ApJL, 673, L215

Otsuji, K., Kitai, R., Ichimoto, K., \& Shibata, K. 2011, PASJ, 63, 1047

Pariat, E., Aulanier, G., Schmieder, B., et al. 2004, ApJ, 614, 1099

Schrijver, C. J. 2009, AdSpR, 43, 739

Shibata, K., Ishido, Y., Acton, L. W., et al. 1992, PASJ, 44, L173

Toriumi, S., \& Yokoyama, T. 2011, ApJ, 735, 126

Tortosa-Andreu, A., \& Moreno-Insertis, F. 2009, A\&A, 507, 949

Valori, G., Green, L. M., Démoulin, P., et al. 2012, SoPh, 278, 73

van Driel-Gesztelyi, L., \& Culhane, J. L. 2009, SSRv, 144, 351

Vargas Domínguez, S., MacTaggart, D., Green, L., van Driel-Gesztelyi, L., \& Hood, A. W. 2012a, SoPh, 278, 33

Vargas Domínguez, S., van Driel-Gesztelyi, L., \& Bellot Rubio, L. R. 2012b, SoPh, 278, 99

Watanabe, H., Kitai, R., Okamoto, K., et al. 2008, ApJ, 684, 736

Yokoyama, T., \& Shibata, K. 1995, Natur, 375, 42

Zwaan, C. 1985, SoPh, 100, 397 